\title{
Detection and investigation of genes with circadian expression pattern in common wheat
}

\author{
Antonina Kiseleva \\ Kurchatov Genomics Center, \\ ICG SB RAS, Novosibirsk, Russia \\ antkiseleva@bionet.nsc.ru
}

\author{
Maria Bragina \\ Kurchatov Genomics Center, \\ ICG SB RAS, Novosibirsk, Russia \\ koltunova@bionet.nsc.ru
}

\author{
Elena Salina \\ Kurchatov Genomics Center, \\ ICG SB RAS, Novosibirsk, Russia \\ salina@bionet.nsc.ru
}

\begin{abstract}
The study of daily oscillations of the wheat transcriptome, the identification of both the core circadian genes and the genes of metabolic pathways under the influence of daily rhythms is the main topic of this work.
\end{abstract}

Keywords - Triticum aestivum, circadian rythms, gene expression

\section{Motivation and aim}

\section{Motivation}

Plant circadian rhythms coordinate such physiological processes as growth, transition to flowering, photosynthesis, response to stress, metabolism and phytohormone signal transduction under the daily and seasonal changes in environmental conditions [1]. Such concurrence has a positive effect on plant growth, development and their adaptability. Genes regulated by circadian rhythms include genes for $\mathrm{CO}_{2}$ assimilation, starch accumulation/degradation in leaves, and storing nutrients (lipids and fatty acids) in seeds [2]. Thus, a large part of the plant transcriptome is controlled by circadian rhythms, which play an important role in the daily regulation of plant physiological processes.

Transcriptome analyses of the circadian mechanisms and its influence on various metabolic processes were performed for such plants as Arabidopsis, Chinese cabbage and soybeans [3]-[5]. The genes of the central oscillator are conservative and their interactions are very similar in different plant species, nevertheless, there are species-specific differences. In addition, in most plant species, the genes of metabolic pathways affected by circadian rhythms are investigated very poorly.

Aim

Common wheat is an allopolyploid (genome AABBDD), so the presence of homoeologous genes in the genome can greatly affect the functioning and regulation of circadian rhythms. Literature analysis and the search in the databases did not find studies exploring the daily expression of the wheat transcriptome, information about the daily expression of the genes and their interaction is practically absent.

\section{Methods}

To identify genes that demonstrate rhythmic changes in expression during the day, we used the Chinese Spring variety transcriptome data obtained at four points during the day $(0$, 3, 9, 16 hours from the moment the light is turned on). Every timepoint was presented by a three biological replicates.

After preprocessing and quality control using the FASTXtoolkit utility the obtained reads were mapped onto the wheat reference transcriptome using Bowtie software. Kallisto v0.42.3 software on default parameters was used to quantifying abundances of transcripts and the trimmed mean of M-values (TMM)-normalized fragments per $\mathrm{kb}$ of Transcripts Per Million (TPM) reads mapped for each transcript. To identify rhythmically expressed genes, we applied the JTK-CYCLE circadian transcript analysis software.

\section{Results}

System analysis of the daily wheat transcriptome let us to identify genes expressed by the circadian type, and metabolic pathways under their control. It was shown, that significant part of the transcriptome is under the control of daily rhythms, and the expression of these genes can vary greatly depending on time. We detected some prominent expression patterns, characterized by peaks in different time points, and described genes underlying these patterns. We analyzed enrichment of gene ontology terms of different patterns and described major metabolic pathways in every group.

\section{Acknowledgment}

The study was funded by RFBR, project No. 20-316-80003.

\section{References}

[1] M. F. Covington, J. N. Maloof, M. Straume, S. A. Kay, and S. L. Harmer, "Global transcriptome analysis reveals circadian regulation of key pathways in plant growth and development", Genome Biol., vol. 9, no. 8, 2008

[2] E. Yakir, D. Hilman, Y. Harir, and R. M. Green, "Regulation of output from the plant circadian clock", FEBS J., vol. 274, no. 2, pp. 335-345, 2007

[3] S. L. Harmer et al., "Orchestrated transcription of key pathways in Arabidopsis by the circadian clock.", Science, vol. 290, no. 5499, pp. 2110-2113, 2000

[4] B. Y. Chow and S. A. Kay, "Global approaches for telling time: omics and the Arabidopsis circadian clock.", Semin. Cell Dev. Biol., vol. 24, no. 5, pp. 383-92, May 2013

[5] J. A. Kim et al., "Transcriptome Analysis of Diurnal Gene Expression in Chinese Cabbage", Genes (Basel)., vol. 10, no. 2, p. 130, 2019 